\title{
VOLTAGE AND CURRENT SPECTRA FOR MATRIX POWER CONVERTERS*
}

\author{
STEPHEN M. $\mathrm{COX}^{\dagger}$ AND STEPHEN C. CREAGH ${ }^{\dagger}$
}

\begin{abstract}
Matrix power converters are used for transforming one alternating-current power supply to another, with different peak voltage and frequency. There are three input lines, with sinusoidally varying voltages which are $120^{\circ}$ out of phase one from another, and the output is to be delivered as a similar three-phase supply. The matrix converter switches rapidly, to connect each output line in sequence to each of the input lines in an attempt to synthesize the prescribed output voltages. The switching is carried out at high frequency and it is of practical importance to know the frequency spectra of the output voltages and of the input and output currents. We determine in this paper these spectra using a new method, which has significant advantages over the prior default method (a multiple Fourier series technique), leading to a considerably more direct calculation. In particular, the determination of the input current spectrum is feasible here, whereas it would be a significantly more daunting procedure using the prior method instead.
\end{abstract}

Key words. matrix power converter, power electronics, Fourier spectrum

AMS subject classifications. 42A16, 94C05

DOI. $10.1137 / 080718863$

1. Introduction. In electrical and electronic engineering, there are many applications in which it is necessary to convert a power supply from one voltage and frequency to another. Particular examples arise in aeronautical and marine applications, since there are increasingly many electrically powered devices aboard aircraft and ships, all with separate demands in terms of power supplies. The field of power conversion, while of great economic importance, thus poses particular technological challenges in aircraft in particular, where it is clearly highly desirable that power conversion be achieved without recourse to heavy bulk energy storage elements.

In modern solid-state power converters, the need for intermediate energy storage is avoided, because the output voltage is generated by rapidly switching between multiple input voltages (see, for example, [7]). The aim is that the low-frequency components of the output synthesize a prescribed waveform, while the high-frequency components related to the switching are ultimately filtered out.

In this paper, we describe a compact means of determining the voltage and current spectra for one such application of particular technological significance: the matrix power converter [12]. This is a device which aims to convert an alternating-current power supply at one voltage and frequency to a second at a different voltage and frequency. Applications of matrix converters include adjustable-speed drives, where the speed of the motor is governed by the frequency of its power supply. A significant benefit of the approach outlined in this paper is that we are able to give explicit and detailed descriptions of input currents, which are considerably more complex and difficult to determine than the output voltages and currents which have been predominantly studied in the past.

* Received by the editors March 19, 2008; accepted for publication (in revised form) December 3, 2008; published electronically February 19, 2009. This work was supported by the Engineering and Physical Sciences Research Council (grant EP/E018580/1).

http://www.siam.org/journals/siap/69-5/71886.html

†School of Mathematical Sciences, University of Nottingham, Nottingham NG7 2RD, UK (stephen.cox@nottingham.ac.uk, stephen.creagh@nottingham.ac.uk). 
The matrix converter switching frequency greatly exceeds the input and output frequencies. The way in which its switching takes place is termed its modulation strategy here, and there are many such strategies adopted in practice, of which a comparatively simple variant is comprehensively analyzed in this paper. The matrix converter successively connects, via switches, each output line to each of the input lines, according to the modulation strategy. Thus the voltage on any given output line comprises short segments of the three input sinusoidal waveforms; it contains both lowfrequency contributions (from the input voltages) and high-frequency contributions (from the switching). It is the spectrum of the output voltages and currents that we compute here, along with the more involved calculation for the corresponding currents drawn from the input lines.

In digital implementations, the input voltages are measured (sampled) at high frequency, at the start of each switching period. Then after each sample a calculation must be done to determine the corresponding switching times to achieve the desired output. This leads to so-called regular or uniform sampling of the input to determine the modulation strategy. The delay between sampling and switching results in undesirable distortion in the form of unintended low-frequency components in the output [5]; it also affects the high-frequency part of the spectrum, but this is not so serious provided that the low-pass filtering still effectively removes such components.

Although less relevant to the power converter application, an alternative sampling technique is also analyzed here: so-called natural sampling, which is widely used in, for example, audio applications $[3,6,8]$. In natural sampling, an analogue device compares one of the input voltages with some reference waveform and switches whenever the two become (instantaneously) equal. The lack of delays in natural sampling leads to a more accurate spectrum for the audio component of the output [7]; a comparison between the spectra for regular and natural sampling allows us to determine what aspects of the former spectrum are due to associated digital implementation effects.

In the engineering literature, spectra for switching devices are generally computed by a multiple Fourier series method usually ascribed to Black [3], but acknowledged to go at least as far back as Bennett [2] (see, for example, [4]). The method involves introducing separate independent variables representing time scaled by each of the input, output, and switching frequencies, then writing the required quantities as multiple Fourier series, in terms of each of these variables separately. The corresponding Fourier coefficients are then computed. Finally, the answer is specialized to the physical case, in which the separate time variables are recognized to be constant multiples of one another. The method is simplest for natural sampling, but can be modified for regular sampling, although it is more algebraically involved in that case.

The major content of this paper is the development of more direct methods than Black's for determining the output voltage, output current, and input current spectra. The methods contained herein can be used for regular and natural sampling, although the order in which various steps are applied is different in the two cases if the greatest efficiency is to be achieved. However, in contrast to Black's method, neither calculation is intrinsically more algebraically cumbersome than the other. Furthermore, our analysis, although presented here for the matrix converter problem, is in fact readily adaptable to any other switching problem for which Black's method is the usual default, for example the modeling of class-D audio amplifiers [6].

In section 2 we outline some notation and describe model calculations (given for both regular and natural sampling), which form the building blocks for many of the subsequent calculations. The output voltages are then computed, using these building-block solutions, for both types of sampling. In section 3 we introduce further 
notation and calculate the output and input currents, for general output impedances, illustrating our results in section 4 . In section 5 we show how to derive more rapidly convergent solutions when the form of the output impedances is known (it is often the case that the output loads may be approximated by a resistor and an inductor in series, for example), considerably reducing the computation time. Our conclusions are given in section 6 . In the appendix, we illustrate how the results in this paper may be extended to a more complicated modulation strategy.

2. Calculation of output voltages. We begin by calculating the spectra of output voltages in a matrix converter. Some of the main ideas of this simpler calculation recur in the more involved calculation for input currents and it is useful to set out the main features and establish notation in the simpler context.

2.1. Notation for voltages. In an idealized matrix converter [12] (see Figure 2.1), there are three input voltages, which we label

$$
v^{A}(t)=\mathrm{e}^{\mathrm{i} \omega_{0} t}, \quad v^{B}(t)=\mathrm{e}^{\mathrm{i}\left(\omega_{0} t+2 \pi / 3\right)}, \quad v^{C}(t)=\mathrm{e}^{\mathrm{i}\left(\omega_{0} t+4 \pi / 3\right)},
$$

where $\omega_{0}$ is the input frequency and voltages are scaled to give unit peak input voltages. Of course, the physical voltages are the real parts of the expressions given in (2.1). It will be convenient to write these collectively as a vector

$$
\mathbf{v}^{\mathrm{in}}(t)=\left(\begin{array}{c}
v^{A}(t) \\
v^{B}(t) \\
v^{C}(t)
\end{array}\right)=\mathrm{e}^{\mathrm{i} \omega_{0} t}\left(\begin{array}{c}
1 \\
p \\
p^{2}
\end{array}\right), \quad \text { where } \quad p=\mathrm{e}^{2 \pi \mathrm{i} / 3} .
$$

Three output voltages, denoted $v^{a}(t), v^{b}(t)$, and $v^{c}(t)$ and written in the vector form,

$$
\mathbf{v}^{\text {out }}(t)=\left(\begin{array}{c}
v^{a}(t) \\
v^{b}(t) \\
v^{c}(t)
\end{array}\right)
$$

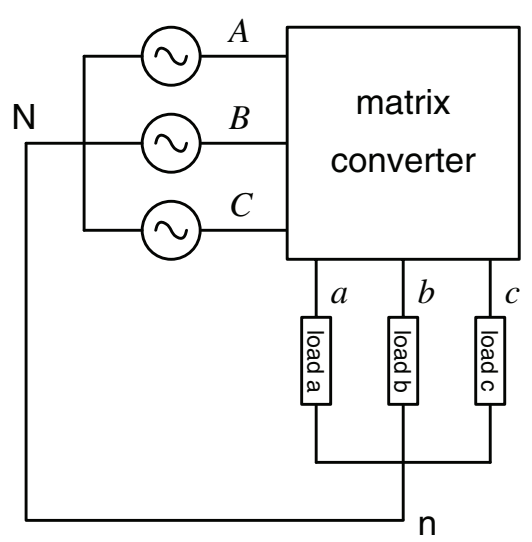

Fig. 2.1. Diagram of a matrix converter. Three input lines ( $A, B$, and $C$ ) each supply sinusoidal voltages, each $120^{\circ}$ out of phase with any other. There are loads on each of the output lines $(a, b$, and $c)$. The neutral point of the input lines is denoted by $\mathrm{N}$ and that of the output lines by $\mathrm{n}$; these neutral points are assumed to be connected to one another, and to be at a nominal zero volts. 
are obtained by sampling the input voltages during intervals which repeat quasiperiodically, according to the modulation strategy. We scale time so that the switching period is unity (hence the switching frequency is $2 \pi$ ), and consider a simple form of switching pattern such that each output, labeled $o=a, b, c$, is of the form [9]

$$
v^{o}(t)= \begin{cases}v^{A}(t) & \text { for } n<t<n+\alpha_{n}^{o}, \\ v^{B}(t) & \text { for } n+\alpha_{n}^{o}<t<n+\beta_{n}^{o}, \\ v^{C}(t) & \text { for } n+\beta_{n}^{o}<t<n+1\end{cases}
$$

(more sophisticated switching strategies can also be analyzed using the methods described in this paper; these are discussed briefly in the appendix). An important assumption in our analysis will be that the switching frequency is much larger than the input frequency: $2 \pi \gg \omega_{0}$. This is certainly the case in practical implementations, to allow the high-frequency switching components to be filtered without affecting the desired low-frequency components.

We encode the relationships in (2.2) using a switching matrix

$$
M(t)=\left(\begin{array}{lll}
F^{a A}(t) & F^{a B}(t) & F^{a C}(t) \\
F^{b A}(t) & F^{b B}(t) & F^{b C}(t) \\
F^{c A}(t) & F^{c B}(t) & F^{c C}(t)
\end{array}\right),
$$

whose elements are either 0 or 1 at any given instant, according to which input and output lines are connected. Then

$$
\mathbf{v}^{\text {out }}(t)=M(t) \mathbf{v}^{\text {in }}(t)
$$

For example,

$$
F^{a A}(t)=\sum_{n=-\infty}^{\infty} \psi_{n, n+\alpha_{n}^{a}}(t)
$$

where the step function $\psi_{t_{1}, t_{2}}$ is defined by

$$
\psi_{t_{1}, t_{2}}(t)= \begin{cases}1 & \text { if } t_{1}<t<t_{2} \\ 0 & \text { otherwise }\end{cases}
$$

and similar expressions can be written for the other elements of $M(t)$, using (2.2).

2.2. A model calculation for the case of uniform sampling. Before describing the full calculation of the three-phase output voltages it is useful to outline a model calculation which illustrates the essence of our approach in a somewhat simpler setting. We consider a function,

$$
F(t)=\sum_{n=-\infty}^{\infty} \psi_{n+\alpha_{n}, n+\beta_{n}}(t),
$$

which samples a unit input voltage over the quasiperiodically repeating intervals

$$
n+\alpha_{n}<t<n+\beta_{n} .
$$

In the case of uniform sampling, we assume that the switching times are determined by sampling the continuous functions

$$
\alpha(\tau)=\mu_{\alpha}+\lambda_{\alpha} \cos \left(\Omega \tau+\delta_{0}\right) \quad \text { and } \quad \beta(\tau)=\mu_{\beta}+\lambda_{\beta} \cos \left(\Omega \tau+\delta_{0}+\delta_{1}\right)
$$


at the evenly spaced times $\tau=n[1,9,10]$. In other words,

$$
\alpha_{n}=\alpha(n) \quad \text { and } \quad \beta_{n}=\beta(n) .
$$

Note that the matrix product in (2.3) consists of sums of functions of the form (2.5), modulated by the harmonic function $\mathrm{e}^{\mathrm{i} \omega_{0} t}$.

Adopting the convention that the Fourier transform is written as

$$
\hat{F}(\omega)=\int_{-\infty}^{\infty} \mathrm{e}^{-\mathrm{i} \omega t} F(t) \mathrm{d} t,
$$

and noting that the Fourier transform of the step function $\psi_{t_{1}, t_{2}}(t)$ is

$$
\hat{\psi}_{t_{1}, t_{2}}(\omega)=\frac{\mathrm{e}^{-\mathrm{i} \omega t_{2}}-\mathrm{e}^{-\mathrm{i} \omega t_{1}}}{-\mathrm{i} \omega}
$$

we find

$$
\hat{F}(\omega)=\sum_{n=-\infty}^{\infty} \mathrm{e}^{-\mathrm{i} n \omega} \hat{\psi}_{\alpha_{n}, \beta_{n}}(\omega)=\sum_{n=-\infty}^{\infty} \mathrm{e}^{-\mathrm{i} n \omega} \frac{\mathrm{e}^{-\mathrm{i} \omega \beta_{n}}-\mathrm{e}^{-\mathrm{i} \omega \alpha_{n}}}{-\mathrm{i} \omega} .
$$

We now make use of the identity [11]

$$
\mathrm{e}^{-\mathrm{i} z \cos \theta}=\sum_{m=-\infty}^{\infty} \mathrm{J}_{m}(z)(-\mathrm{i})^{m} \mathrm{e}^{\mathrm{i} m \theta}
$$

so that

$$
\frac{\mathrm{e}^{-\mathrm{i} \omega \beta_{n}}-\mathrm{e}^{-\mathrm{i} \omega \alpha_{n}}}{-\mathrm{i} \omega}=\sum_{m=-\infty}^{\infty} X_{m}(\omega) \mathrm{e}^{\mathrm{i} n m \Omega+\mathrm{i} m \delta_{0}}
$$

where, using (2.6), we find

$$
X_{m}(\omega)=\frac{(-\mathrm{i})^{m}}{-\mathrm{i} \omega}\left(\mathrm{e}^{-\mathrm{i} \omega \mu_{\beta}+\mathrm{i} m \delta_{1}} \mathrm{~J}_{m}\left(\omega \lambda_{\beta}\right)-\mathrm{e}^{-\mathrm{i} \omega \mu_{\alpha}} \mathrm{J}_{m}\left(\omega \lambda_{\alpha}\right)\right) .
$$

It is useful to record the following limiting values:

$$
X_{m}(0)= \begin{cases}\mu_{\beta}-\mu_{\alpha} & \text { when } m=0, \\ \frac{1}{2}\left(\mathrm{e}^{m \mathrm{i} \delta_{1}} \lambda_{\beta}-\lambda_{\alpha}\right) & \text { when } m= \pm 1, \\ 0 & \text { otherwise. }\end{cases}
$$

Then, using the Poisson summation formula in the form

$$
\sum_{n=-\infty}^{\infty} \mathrm{e}^{\mathrm{i} n(\omega-m \Omega)}=2 \pi \sum_{n=-\infty}^{\infty} \delta(\omega-m \Omega-2 \pi n),
$$

we find that

$$
\begin{aligned}
\hat{F}(\omega) & =\sum_{n=-\infty}^{\infty} \sum_{m=-\infty}^{\infty} X_{m}(\omega) \mathrm{e}^{-\mathrm{i} n(\omega-m \Omega)+\mathrm{i} m \delta_{0}} \\
& =2 \pi \sum_{n=-\infty}^{\infty} \sum_{m=-\infty}^{\infty} X_{m}\left(\omega_{n m}\right) \mathrm{e}^{m \mathrm{i} \delta_{0}} \delta\left(\omega-\omega_{n m}\right)
\end{aligned}
$$


where we denote

$$
\omega_{n m}=2 \pi n+m \Omega
$$

The result (2.11) can alternatively be written in the time domain as

$$
F(t)=\sum_{n=-\infty}^{\infty} \sum_{m=-\infty}^{\infty} X_{m}\left(\omega_{n m}\right) \mathrm{e}^{\mathrm{i} \omega_{n m} t+\mathrm{i} m \delta_{0}}
$$

We therefore find that the spectrum of the function $F(t)$ is confined to integer combinations of the switching frequency $2 \pi$ and the modulation frequency $\Omega$ (cf. [3]). Furthermore, the frequencies of particular practical interest are those with $n=0$ (those with $n \neq 0$ will be filtered out), and it is easily verified that in the limit $\Omega \ll 2 \pi$ the dominant frequencies with $n=0$ are $\omega_{00}=0$ and $\omega_{0 \pm 1}= \pm \Omega$. It will later prove useful to denote by $F_{0}(t)$ the terms in (2.13) for $n=0$; summing over $m$ the contributions to $F(t)$ with $n=0$ is then easily seen to give

$$
F_{0}(t) \equiv \sum_{m=-\infty}^{\infty} X_{m}\left(\omega_{0 m}\right) \mathrm{e}^{\mathrm{i} \omega_{0 m} t+\mathrm{i} m \delta_{0}}=\beta(t)-\alpha(t)+O(\Omega)
$$

By comparing (2.14) with (2.6), we see that the $n=0$ contribution $F_{0}(t)$ is thus, with errors of order $\Omega$, a sinusoidal signal with frequency $\Omega$, plus a constant signal.

2.3. Output voltages in the case of uniform sampling. The model calculation in section 2.2 can now be used as the basis for a more complete description of the output voltages. We begin by describing more explicitly the switching conventions in (2.2). These are designed to generate output voltages

$$
\mathbf{v}_{\mathrm{ref}}^{\mathrm{out}}(t)=\left(\begin{array}{c}
v_{\mathrm{ref}}^{a}(t) \\
v_{\text {ref }}^{b}(t) \\
v_{\text {ref }}^{c}(t)
\end{array}\right)=q \mathrm{e}^{\mathrm{i} \omega_{1} t}\left(\begin{array}{c}
1 \\
p \\
p^{2}
\end{array}\right),
$$

where $\omega_{1}$ is the output frequency and $q$ is the output amplitude. The subscript "ref" indicates that the corresponding quantity is the intended, reference state; the actual output voltage will generally approximate this reference value in its low-frequency spectrum, but also contain slight low-frequency distortion terms and significant highfrequency components. The matching of the low-frequency terms in $\mathbf{v}^{\text {out }}(t)$ and $\mathbf{v}_{\text {ref }}^{\text {out }}(t)$ is achieved by letting $\alpha_{n}^{o}$ and $\beta_{n}^{o}$ in (2.2) oscillate with an appropriate frequency that is much smaller than the switching frequency.

We focus on the simplest Venturini switching $[1,9,10]$, in which, for each output $o=a, b$, or $c$, the times $\alpha_{n}^{o}$ and $\beta_{n}^{o}$ are obtained by sampling smooth functions as in (2.6) and (2.7), with

$$
\alpha^{o}(\tau)=\frac{1}{3}+\frac{2}{3} q \cos \left(\Omega \tau+\delta^{o}\right), \quad \beta^{o}(\tau)=\frac{2}{3}+\frac{2}{3} q \cos \left(\Omega \tau+\delta^{o}-\frac{\pi}{3}\right) .
$$

Here,

$$
\Omega \equiv \omega_{1}-\omega_{0}
$$

is the difference between output and input frequencies and

$$
\delta^{a}=0, \quad \delta^{b}=\frac{2}{3} \pi, \quad \text { and } \quad \delta^{c}=\frac{4}{3} \pi .
$$


The coefficients in (2.16) were originally derived by attempting to generate the correct low-frequency components in the output voltages, in the limit $\Omega \rightarrow 0$.

We now adapt the model calculation in section 2.2 to describe the output voltages. Note that (2.3) indicates that any given output voltage can be written as

$$
\begin{aligned}
v^{o}(t) & =\mathrm{e}^{\mathrm{i} \omega_{0} t} \sum_{n=-\infty}^{\infty} \psi_{n, n+\alpha_{n}^{o}}(t)+p \psi_{n+\alpha_{n}^{o}, n+\beta_{n}^{o}}(t)+p^{2} \psi_{n+\beta_{n}^{o}, n+1}(t) \\
& =\left(F^{o A}(t)+p F^{o B}(t)+p^{2} F^{o C}(t)\right) \mathrm{e}^{\mathrm{i} \omega_{0} t}, \quad o=a, b, c,
\end{aligned}
$$

where

$$
\begin{aligned}
& F^{o A}(t)=\sum_{n=-\infty}^{\infty} \psi_{n, n+\alpha_{n}^{o}}(t), \\
& F^{o B}(t)=\sum_{n=-\infty}^{\infty} \psi_{n+\alpha_{n}^{o}, n+\beta_{n}^{o}}(t), \\
& F^{o C}(t)=\sum_{n=-\infty}^{\infty} \psi_{n+\beta_{n}^{o}, n+1}(t)
\end{aligned}
$$

are all particular cases of the function $F(t)$ described in section 2.2. Repeating the calculations there, we find that $F^{o A}(t), F^{o B}(t)$, and $F^{o C}(t)$ have Fourier transforms

$$
\hat{F}^{o i}(\omega)=2 \pi \sum_{n=-\infty}^{\infty} \sum_{m=-\infty}^{\infty} X_{m}^{i}\left(\omega_{n m}\right) \mathrm{e}^{m \mathrm{i} \delta^{o}} \delta\left(\omega-\omega_{n m}\right), \quad i=A, B, C,
$$

where

$$
\begin{aligned}
& X_{m}^{A}(\omega)=\frac{(-\mathrm{i})^{m}}{-\mathrm{i} \omega}\left[\mathrm{e}^{-\mathrm{i} \omega / 3} \mathrm{~J}_{m}\left(\frac{2}{3} q \omega\right)-\delta_{m 0}\right], \\
& X_{m}^{B}(\omega)=\frac{(-\mathrm{i})^{m}}{-\mathrm{i} \omega}\left[\mathrm{e}^{-2 \mathrm{i} \omega / 3-\mathrm{i} m \pi / 3}-\mathrm{e}^{-\mathrm{i} \omega / 3}\right] \mathrm{J}_{m}\left(\frac{2}{3} q \omega\right), \\
& X_{m}^{C}(\omega)=\frac{(-\mathrm{i})^{m}}{-\mathrm{i} \omega}\left[\mathrm{e}^{-\mathrm{i} \omega} \delta_{m 0}-\mathrm{e}^{-2 \mathrm{i} \omega / 3-\mathrm{i} m \pi / 3} \mathrm{~J}_{m}\left(\frac{2}{3} q \omega\right)\right] ;
\end{aligned}
$$

here $\delta_{0 m}$ is the Kronecker $\delta$. Note that the quantities $X_{m}^{i}(\omega)$ do not depend on the output line.

The transformed output voltages are given by

$$
\hat{v}^{o}(\omega)=\hat{F}^{o A}\left(\omega-\omega_{0}\right)+p \hat{F}^{o B}\left(\omega-\omega_{0}\right)+p^{2} \hat{F}^{o C}\left(\omega-\omega_{0}\right),
$$

which can then be written as

$$
\hat{v}^{o}(\omega)=2 \pi \sum_{n=-\infty}^{\infty} \sum_{m=-\infty}^{\infty} \mathrm{e}^{\mathrm{i} m \delta^{\circ}} V_{n m} \delta\left(\omega-\Omega_{n m}\right),
$$

where we denote

$$
V_{n m}=X_{m}^{A}\left(\omega_{n m}\right)+p X_{m}^{B}\left(\omega_{n m}\right)+p^{2} X_{m}^{C}\left(\omega_{n m}\right)
$$

and

$$
\Omega_{n m}=\omega_{0}+\omega_{n m}=\omega_{0}+2 \pi n+m \Omega .
$$


The corresponding expression in the time domain gives us the key result of this section, that the output voltages may be written as

$$
v^{o}(t)=\sum_{n=-\infty}^{\infty} \sum_{m=-\infty}^{\infty} V_{n m} \mathrm{e}^{\mathrm{i} \Omega_{n m} t+\mathrm{i} m \delta^{o}} .
$$

Note that the amplitudes $V_{n m}$ are common to all three output lines; the differences between the three output voltages result from the different values of $\delta^{o}$ for $o=a, b, c$ in $(2.20)$.

The most physically interesting part of the result is

$$
v_{0}^{o}(t) \equiv \sum_{m=-\infty}^{\infty} V_{0 m} \mathrm{e}^{i \Omega_{0 m} t+\mathrm{i} m \delta^{o}}=\sum_{m=-1}^{1} V_{0 m} \mathrm{e}^{i \Omega_{0 m} t+\mathrm{i} m \delta^{o}}+O(\Omega) .
$$

In analogy with (2.10), we find the limiting cases

$$
X_{0}^{i}(0)=\frac{1}{3}, \quad i=A, B, C
$$

and

$$
X_{ \pm 1}^{A}(0)=\frac{1}{3} q, \quad X_{ \pm 1}^{B}(0)=\frac{1}{3} q\left(\mathrm{e}^{\mp \mathrm{i} \pi / 3}-1\right), \quad X_{ \pm 1}^{C}(0)=-\frac{1}{3} q \mathrm{e}^{\mp \mathrm{i} \pi / 3},
$$

and these can be used to show that the rightmost sum in (2.21) approximately returns the voltages required in $(2.15)$, so that

$$
v_{0}^{o}(t)=v_{\text {ref }}^{o}(t)+O(\Omega) .
$$

The $O(\Omega)$ error results from the discrete sampling of the input voltages used to compute the modulation strategy with regular sampling. It can be eliminated by changing the modulation strategy to natural sampling, which is now described.

2.4. A model calculation for the case of natural sampling. We now turn to the case of natural sampling. Here, in contrast to regular sampling, the input voltages are monitored continuously, and switching takes place at the instants when these voltages become equal to some other reference voltage. Because of the need to continuously monitor the input voltages, such sampling is generally implemented using analogue electronics. The key additional algebraic complication associated with natural sampling is that the switching times satisfy implicit equations. To analyze natural sampling, then, we consider once again the model sum in (2.5) except that the switching times are chosen to satisfy conditions

$$
\alpha_{n}=A\left(n+\alpha_{n}\right) \quad \text { and } \quad \beta_{n}=B\left(n+\beta_{n}\right),
$$

where $A(t)=\mu_{\alpha}+\lambda_{\alpha} \cos \left(\Omega t+\delta_{0}\right)$ and $B(t)=\mu_{\beta}+\lambda_{\beta} \cos \left(\Omega t+\delta_{0}+\delta_{1}\right)$.

We note that, according to (2.24), we may consider $\alpha_{n}$ and $\beta_{n}$ to be irregular samples of the continuous functions $A(t)$ and $B(t)$. However, to make analytical headway with our approach, it is preferable instead to regard $\alpha_{n}$ and $\beta_{n}$ as being obtained by regularly sampling continuous functions $\alpha(\tau)$ and $\beta(\tau)$ as in (2.7). Now, however, it is the functions $A(t)$ and $B(t)$ that are prescribed explicitly while the functions $\alpha(\tau)$ and $\beta(\tau)$ are determined implicitly by

$$
\alpha(\tau)=A(\tau+\alpha(\tau)) \quad \text { and } \quad \beta(\tau)=B(\tau+\beta(\tau)),
$$


which are continuous versions of (2.24).

In the case of natural sampling, the output spectrum is best calculated by performing Poisson resummation before taking the Fourier transform of the function $F(t)$. Using the version

$$
\sum_{n=-\infty}^{\infty} f(n)=\sum_{n=-\infty}^{\infty} \int_{-\infty}^{\infty} \mathrm{e}^{2 \pi n \mathrm{i} \tau} f(\tau) \mathrm{d} \tau
$$

of Poisson resummation on expression (2.5) for $F(t)$, we find that

$$
F(t)=\sum_{n=-\infty}^{\infty} \int_{-\infty}^{\infty} \mathrm{e}^{2 \pi n \mathrm{i} \tau} \psi_{\tau+\alpha(\tau), \tau+\beta(\tau)}(t) \mathrm{d} \tau=\sum_{n=-\infty}^{\infty} \int_{\tau_{B}(t)}^{\tau_{A}(t)} \mathrm{e}^{2 \pi n \mathrm{i} \tau} \mathrm{d} \tau,
$$

where $\tau_{B}(t)$ and $\tau_{A}(t)$ are, respectively, the values of $\tau$ at which the step function $\psi_{\tau+\alpha(\tau), \tau+\beta(\tau)}(t)$ switches on and then off again for fixed $t$. These switching times satisfy the conditions $t=\tau_{A}+\alpha\left(\tau_{A}\right)$ and $t=\tau_{B}+\beta\left(\tau_{B}\right)$, which can be rearranged to give

$$
\tau_{A}(t)=t-\alpha\left(\tau_{A}\right)=t-A(t) \quad \text { and } \quad \tau_{B}(t)=t-\beta\left(\tau_{B}\right)=t-B(t) .
$$

We therefore have

$$
F(t)=\sum_{n=-\infty}^{\infty} \int_{t-B(t)}^{t-A(t)} \mathrm{e}^{2 \pi n \mathrm{i} \tau} \mathrm{d} \tau=\sum_{n=-\infty}^{\infty} \mathrm{e}^{2 \pi n \mathrm{i} t} F_{n}(t),
$$

where

$$
F_{n}(t)=\int_{-B(t)}^{-A(t)} \mathrm{e}^{2 \pi n \mathrm{i} \tau} \mathrm{d} \tau= \begin{cases}B(t)-A(t) & \text { if } n=0, \\ \frac{\mathrm{e}^{-2 \pi n \mathrm{i} B(t)}-\mathrm{e}^{-2 \pi n \mathrm{i} A(t)}}{-2 \pi n \mathrm{i}} & \text { otherwise. }\end{cases}
$$

Equation (2.8) now gives

$$
F(t)=\sum_{n=-\infty}^{\infty} \sum_{m=-\infty}^{\infty} X_{m}(2 \pi n) \mathrm{e}^{\mathrm{i} \omega_{n m} t+\mathrm{i} m \delta^{\circ}},
$$

where $X_{m}(\omega)$ has been defined in (2.9) and, in the special case $n=0$, we may use $(2.10) ; \omega_{n m}$ has been defined in (2.12). The Fourier transform is

$$
\hat{F}(\omega)=2 \pi \sum_{n=-\infty}^{\infty} \sum_{m=-\infty}^{\infty} X_{m}(2 \pi n) \mathrm{e}^{m \mathrm{i} \delta^{o}} \delta\left(\omega-\omega_{n m}\right) .
$$

These results are similar to those given in section 2.2 for the case of uniform sampling, except that the amplitude functions $X_{m}$ are evaluated at different values of the argument $\left(2 \pi n\right.$ here, rather than $\omega_{n m}$ for uniform sampling). This difference has a dramatic effect on the terms with $n=0$, however, which collectively contribute

$$
F_{0}(t)=\sum_{m=-\infty}^{\infty} X_{m}(0) \mathrm{e}^{\mathrm{i} \omega_{0 m} t+\mathrm{i} m \delta^{o}}=B(t)-A(t)
$$

to the sum (see also (2.28)). This is the natural-sampling analogue of (2.14) and it is exact, which means that the only low frequencies present in $F(t)$ are those present in the prescribed functions $A(t)$ and $B(t)$ (cf. $[3,6,8]$ ). 
2.5. Output voltages in the case of natural sampling. We now adapt the model calculation in section 2.4 to the case of three-phase output voltages produced by naturally sampling input voltages as in (2.2). The difference from the calculation in section 2.3 is that here the switching times are determined implicitly by equations of the form

$$
\alpha_{n}^{o}=A^{o}\left(n+\alpha_{n}^{o}\right) \quad \text { and } \quad \beta_{n}^{o}=B^{o}\left(n+\beta_{n}^{o}\right),
$$

where

$$
A^{o}(\tau)=\frac{1}{3}+\frac{2}{3} q \cos \left(\Omega \tau+\delta^{o}\right), \quad B^{o}(\tau)=\frac{2}{3}+\frac{2}{3} q \cos \left(\Omega \tau+\delta^{o}-\frac{\pi}{3}\right),
$$

rather than being given directly as in (2.16).

There is nothing fundamentally new in the calculation here that has not already been covered in sections 2.3 and 2.4 so we simply present the main results. The output voltages can be given in the form

$$
v^{o}(t)=\sum_{n=-\infty}^{\infty} \sum_{m=-\infty}^{\infty} \tilde{V}_{n m} \mathrm{e}^{i \Omega_{n m} t+\mathrm{i} m \delta^{o}},
$$

which is similar to (2.20), except that in the expression

$$
\tilde{V}_{n m}=X_{m}^{A}(2 \pi n)+p X_{m}^{B}(2 \pi n)+p^{2} X_{m}^{C}(2 \pi n),
$$

the arguments of the functions $X_{m}^{i}$ (which are once again given by (2.19)) are $2 \pi n$ instead of $\omega_{n m}$. The part of $v^{o}(t)$ of most physical interest is the contribution from terms with $n=0$; this contribution can be written, using (2.10),

$$
v_{0}^{o}(t)=\sum_{m=-1}^{1}\left(X_{m}^{A}(0)+p X_{m}^{B}(0)+p^{2} X_{m}^{C}(0)\right) \mathrm{e}^{i \Omega_{0 m} t+\mathrm{i} m \delta^{o}}=q \mathrm{e}^{\mathrm{i} \omega_{1} t+\mathrm{i} \delta^{o}} .
$$

Remarkably, this result coincides exactly with the desired form in (2.15). We emphasize that the result $v_{0}^{o}(t)=v_{\text {ref }}^{o}(t)$ is exact for natural sampling (cf. the corresponding result (2.23) for uniform sampling, where there are errors of order $\Omega$ ). In other contexts, this exact capture of some reference output is well known $[3,6,7,8]$.

3. Input and output currents. We now turn our attention to the currents in the system. The output currents are readily determined from the output voltages, provided that the output impedances are known (for simplicity, we suppose that the output neutral is connected to the supply neutral - see Figure 2.1). In order to construct the input currents, however, we must examine how the modulation strategy assigns the output currents to each input line; we are thus led to consider two separate discrete sampling processes, and the Fourier transform is as a result more complex to analyze. We note that in practice the input currents are monitored to provide a diagnostic of the system, and thus a knowledge of their spectrum is of particular practical utility.

The general discussion below applies equally to either regular or natural sampling. In section 3.4 below, we specialize the analysis to the two cases separately.

3.1. Notation for currents. We begin by setting out notation, building on the discussion in section 2.1. We adopt similar conventions for the input and output currents, writing 


$$
\mathbf{i}^{\text {in }}(t)=\left(i^{A}, i^{B}, i^{C}\right)^{T} \quad \text { and } \quad \mathbf{i}^{\text {out }}(t)=\left(i^{a}, i^{b}, i^{c}\right)^{T},
$$

where the superscript $T$ denotes the transpose, and we note that current conservation means that these are connected by the transpose of the switching matrix [12], according to

$$
\mathbf{i}^{\text {in }}(t)=M(t)^{T} \mathbf{i}^{\text {out }}(t) .
$$

A central goal in this paper is to compute the spectrum of the input currents in terms of the input voltages, and there are two elements to this calculation: summing over the windows of time in which the input voltage is sampled as a simple harmonic, and calculating the contributions from individual windows within that sum.

The summation is a double sum arising from the combined matrix products in (2.3) and (3.1) and is described explicitly below. We first describe in general terms the contribution of an individual element in this sum.

\subsection{Loading the output: Currents associated with individual input pulses. Let}

$$
v_{t_{3}, t_{4}}(t)=\psi_{t_{3}, t_{4}}(t) \mathrm{e}^{i \omega_{0} t}
$$

represent an output voltage obtained by sampling a harmonic input voltage $\mathrm{e}^{i \omega_{0} t}$ over the window $t_{3}<t<t_{4}$. Let the output be connected to a load described by the impedance $Z(\omega)$, so that in the frequency domain the output current is

$$
\hat{\imath}_{t_{3}, t_{4}}(\omega)=\frac{1}{Z(\omega)} \hat{v}_{t_{3}, t_{4}}(\omega)=\frac{1}{Z(\omega)} \hat{\psi}_{t_{3}, t_{4}}\left(\omega-\omega_{0}\right) .
$$

We describe the corresponding relation in the time domain using an admittance operator $Y$, such that $i_{t_{3}, t_{4}}(t)=Y v_{t_{3}, t_{4}}(t)$. This will describe an output current that switches on at $t=t_{3}$, is driven harmonically in the window $t_{3}<t<t_{4}$, and decays as a transient thereafter, when $t_{4}<t<\infty$. If a given input line connects to the output in question during the window $t_{1}<t<t_{2}$, then we denote the corresponding contribution to that input current by

$$
i_{t_{1}, t_{2}, t_{3}, t_{4}}(t)=\psi_{t_{1}, t_{2}}(t) i_{t_{3}, t_{4}}(t)=\psi_{t_{1}, t_{2}}(t) Y v_{t_{3}, t_{4}}(t) .
$$

The corresponding relation in the frequency domain is

$$
\hat{\imath}_{t_{1}, t_{2}, t_{3}, t_{4}}(\omega)=\frac{1}{2 \pi} \hat{\psi}_{t_{1}, t_{2}}(\omega) * \hat{\imath}_{t_{3}, t_{4}}(\omega)=\frac{1}{2 \pi} \hat{\psi}_{t_{1}, t_{2}}(\omega) *\left[\frac{1}{Z(\omega)} \hat{v}_{t_{3}, t_{4}}(\omega)\right],
$$

where $*$ denotes convolution.

3.3. Loading the output: Total currents. Net input currents are obtained by summing individual contributions of the form (3.4), as governed by the matrix products in (2.3) and (3.1). We now outline details and notation for this process. Let the output voltages and currents be related by

$$
\mathbf{i}^{\text {out }}(t)=\mathcal{Y} \mathbf{v}^{\text {out }}(t)
$$

where $\mathcal{Y}$ is the diagonal matrix of admittance operators

$$
\mathcal{Y}=\left(\begin{array}{ccc}
Y^{a} & 0 & 0 \\
0 & Y^{b} & 0 \\
0 & 0 & Y^{c}
\end{array}\right) \equiv \operatorname{diag}\left(Y^{a}, Y^{b}, Y^{c}\right),
$$


and where the diagonal elements are specific to each output. In Fourier representation, the admittance operator is represented by the simple diagonal matrix

$$
\mathcal{Y}(\omega)=\operatorname{diag}\left(Z^{a}(\omega)^{-1}, Z^{b}(\omega)^{-1}, Z^{c}(\omega)^{-1}\right)
$$

of output-specific admittances. Combining (3.5) with (2.3) and (3.1), we may write

$$
\mathbf{i}^{\text {in }}(t)=M(t)^{T} \mathcal{Y} M(t) \mathbf{v}^{\text {in }}(t) .
$$

Let us denote by

$$
Q(t)=M(t)^{T} \mathcal{Y} M(t) \mathrm{e}^{i \omega_{0} t}
$$

the combined operator relating the input currents to the (known) input voltages, so that

$$
\mathbf{i}^{\mathrm{in}}(t)=Q(t)\left(\begin{array}{c}
1 \\
p \\
p^{2}
\end{array}\right)
$$

A typical element of $Q(t)$ can be written as a simple sum over outputs. Specifically,

$$
Q^{i j}(t)=\sum_{o=a, b, c} F^{o i}(t) Y^{o} F^{o j}(t) \mathrm{e}^{i \omega_{0} t}
$$

where the row index $i=A, B, C$ and the column index $j=A, B, C$ are labels of inputs. Each switching element $F^{o i}(t)$ is, in fact, a train of step-functions, as illustrated in (2.4). This allows us to write more explicitly, for example,

$$
Q^{B B}(t)=\sum_{o=a, b, c} \sum_{m=-\infty}^{\infty} \sum_{n=-\infty}^{\infty} \psi_{m+\alpha_{m}^{o}, m+\beta_{m}^{o}}(t) Y^{o} \psi_{n+\alpha_{n}^{o}, n+\beta_{n}^{o}}(t) \mathrm{e}^{i \omega_{0} t}
$$

Note that the individual terms in this sum are of the form given in (3.3), with $t_{1}=m+\alpha_{m}^{o}, t_{2}=m+\beta_{m}^{o}, t_{3}=n+\alpha_{n}^{o}$, and $t_{4}=n+\beta_{n}^{o}$. Other entries in the matrix $Q(t)$ can be written similarly, except that alternative combinations of switching times are substituted for $t_{1}, t_{2}, t_{3}$, and $t_{4}$.

In the frequency domain, a typical element of the matrix $\hat{Q}(\omega)$ can be written, in analogy with (3.7),

$$
\hat{Q}^{i j}(\omega)=\frac{1}{2 \pi} \sum_{o=a, b, c} \hat{F}^{o i}(\omega) *\left[\frac{1}{Z^{o}(\omega)} \hat{F}^{o j}\left(\omega-\omega_{0}\right)\right] .
$$

3.4. Direct calculation of input currents. We now outline a direct calculation of the input currents, for regular or natural sampling, using (3.8) and (3.9), respectively, as a basis. Nothing is assumed here about the form of the output impedances and the method is very general. More efficient, but less general, methods are described later, for specific forms of the output impedances. The difference between the two sets of calculations derives from whether we perform the convolution integral in (3.9) before or after the double sum over switching times. In this section, the sum is performed first and the convolution after. 
3.4.1. Regular sampling. We first describe the calculation for regular sampling. For the contribution $\hat{Q}^{i j}(\omega)$, as described by (3.9), we first write

$$
\hat{Q}^{i j}(\omega)=\sum_{o=a, b, c} \hat{Q}^{o, i j}(\omega)
$$

where

$$
\hat{Q}^{o, i j}(\omega)=\frac{1}{2 \pi} \hat{F}^{o i}(\omega) *\left[\frac{1}{Z^{o}(\omega)} \hat{F}^{o j}\left(\omega-\omega_{0}\right)\right],
$$

and the function $\hat{F}^{o i}(\omega)$ has been defined in (2.17). In this expression the function

$$
\hat{F}^{o i}(\omega)=2 \pi \sum_{n=-\infty}^{\infty} \sum_{m=-\infty}^{\infty} X_{m}^{i}\left(\omega_{n m}\right) \mathrm{e}^{m \mathrm{i} \delta^{o}} \delta\left(\omega-\omega_{m n}\right)
$$

is convolved with

$$
\frac{1}{Z^{o}(\omega)} \hat{F}^{o j}\left(\omega-\omega_{0}\right)=2 \pi \sum_{n=-\infty}^{\infty} \sum_{m=-\infty}^{\infty} \frac{X_{m}^{j}\left(\omega_{n m}\right) \mathrm{e}^{m \mathrm{i} \delta^{o}}}{Z^{o}\left(\omega_{0}+\omega_{m n}\right)} \delta\left(\omega-\omega_{0}-\omega_{m n}\right),
$$

and the result is a quadruple sum

$$
\hat{Q}^{o, i j}(\omega)=2 \pi \sum_{k l n m} W_{k l n m}^{o, i j} \delta\left(\omega-\omega_{0}-\omega_{k l}-\omega_{n m}\right),
$$

where

$$
W_{k l n m}^{o, i j}=X_{l}^{i}\left(\omega_{k l}\right) X_{m}^{j}\left(\omega_{n m}\right)\left[\frac{\mathrm{e}^{\mathrm{i}(l+m) \delta^{o}}}{Z^{o}\left(\omega_{0}+\omega_{n m}\right)}\right]
$$

and, in the sum, the indices $k, l, n$, and $m$ run independently from $-\infty$ to $\infty$. The output-dependent parts (to be summed over later) have been isolated within square brackets in this expression. Using the fact that $\omega_{k l}+\omega_{n m}=\omega_{k+n, l+m}$, this result can alternatively be stated in the time domain as

$$
Q^{o, i j}(t)=\sum_{k l n m} W_{k l n m}^{o, i j} \mathrm{e}^{\mathrm{i}\left(\omega_{0}+\omega_{k+n, l+m}\right) t} .
$$

It is convenient to group terms in this sum with a common frequency, giving

$$
Q^{o, i j}(t)=\sum_{N M} \mathcal{Q}_{N M}^{o, i j} \mathrm{e}^{\mathrm{i} \Omega_{N M} t},
$$

where $\Omega_{N M}=\omega_{0}+\omega_{N M}=\omega_{0}+2 \pi N+M \Omega$ and

$$
\mathcal{Q}_{N M}^{o, i j}=\sum_{n m} W_{N-n, M-m, n, m}^{o, i j} .
$$

In this result, the amplitude $\mathcal{Q}_{N M}^{o, i j}$ of a term with a given frequency $\Omega_{N M}$ is expressed as a double sum. Finally, we note that, according to (3.6), the total current in an input labeled by the superscript $i$ can be obtained from the results above using

$$
i^{i}(t)=\sum_{o=a, b, c} Q^{o, i A}(t)+p Q^{o, i B}(t)+p^{2} Q^{o, i C}(t) .
$$

Although these formulas for the input currents seem rather unwieldy, we may already note the potentially diagnostically useful result that, if all outputs have equal impedance, then the sum over outputs produces a factor $\sum_{o} \mathrm{e}^{M \mathrm{i} \delta^{\circ}}=1+\mathrm{e}^{2 \pi M \mathrm{i} / 3}+$ $\mathrm{e}^{4 \pi M \mathrm{i} / 3}$, which vanishes unless $M$ is a multiple of 3 . Hence, in this special case, the frequencies $\Omega_{N M}=\omega_{0}+2 \pi N+M \Omega$ appear only where $M$ is a multiple of 3 . 
3.4.2. Natural sampling. We may readily adapt the expressions derived in the previous section to the case of natural sampling, without repeating the entire calculation. To do so, we simply note, from (2.18) and (2.29), that the only material difference in the natural sampling case lies in the arguments of the functions $X_{m}^{i}$ used in the definitions of $\hat{F}^{o i}(\omega)$. Hence the input currents are still given by an expression of the form (3.13), with $Q^{o, i j}$ given by (3.11); however, $\mathcal{Q}_{N M}^{o, i j}$ is now given by

$$
\mathcal{Q}_{N M}^{o, i j}=\sum_{n m} X_{M-m}^{i}(2 \pi(N-n)) X_{m}^{j}(2 \pi n)\left[\frac{\mathrm{e}^{\mathrm{i} M \delta^{o}}}{Z^{o}\left(\omega_{0}+\omega_{n m}\right)}\right],
$$

rather than by (3.12). It is interesting to note that in the case of input currents, unlike in the cases of output voltages and currents, natural sampling does not produce a clean single harmonic when the high-frequency terms with $N \neq 0$ are filtered out.

4. Results for the voltage and current spectra. In this section we illustrate the results above for the output voltage and input current spectra. Recalling that the switching frequency has been scaled to $2 \pi$, we choose parameter values as follows:

$$
\omega_{0}=\frac{1}{20} \times 2 \pi, \quad \omega_{1}=\frac{1}{25} \times 2 \pi, \quad q=0.4 .
$$

The corresponding output voltage spectrum is independent of the output loads, and is shown in Figure 4.1, for regular and natural sampling. The primary feature of note is the exact reproduction of the low-frequency $(n=0)$ part of the voltage spectrum for natural sampling and, in contrast, the significant low-frequency distortion introduced by regular sampling.
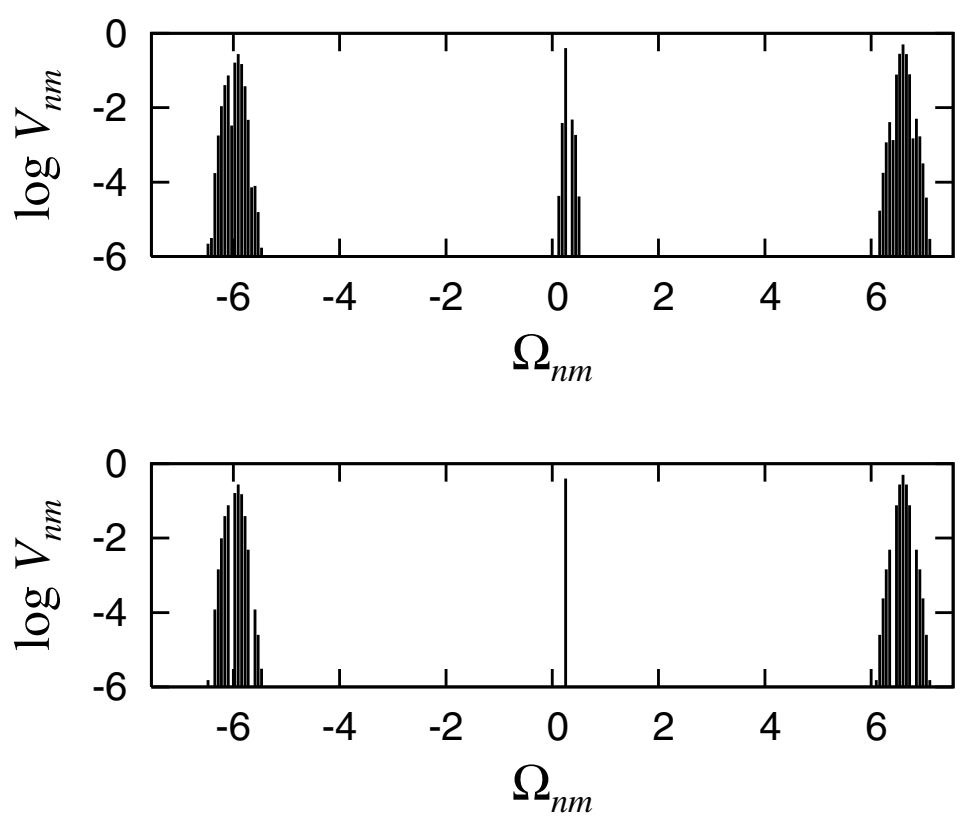

FIG. 4.1. Spectrum of the output voltages, for parameter values (4.1). Upper plot: regular sampling. Lower plot: natural sampling. Note the significant low-frequency distortion of the regularly sampled case. 

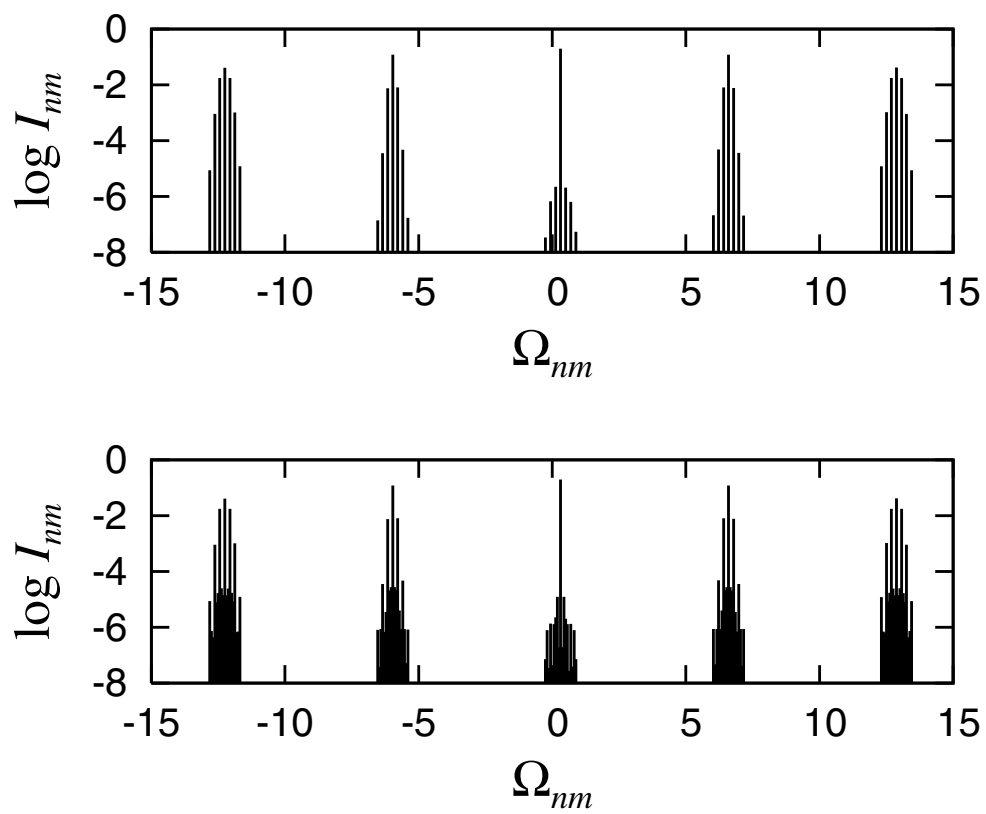

FIG. 4.2. Spectrum of the input currents in line A with regular sampling, for parameter values (4.1). Upper plot: balanced loads, with (4.3). Lower plot: unbalanced loads, with (4.4). Spectra for input lines $B$ and $C$ are similar.

To determine the input currents, we must specify the output loads, which will each comprise a resistor and an inductor in series, so that

$$
Z^{o}(\omega)=R^{o}+\mathrm{i} \omega L^{o}, \quad o=a, b, \text { or } c .
$$

We consider two cases. In the first, all output lines offer equal impedance, with

$$
R^{a}=R^{b}=R^{c}=5 \Omega, \quad L^{a}=L^{b}=L^{c}=5 \mathrm{mH} ;
$$

in the second, the output impedances are unbalanced, and we take

$$
R^{a}=R^{b}=R^{c}=5 \Omega, \quad L^{a}=L^{b}=5 \mathrm{mH}, \quad L^{c}=0 \mathrm{mH} .
$$

From Figure 4.2 we see that the frequency spectrum for the input current is sparser for the balanced load. In fact, as argued above, the spectrum is confined to frequencies of the form $\omega_{0}+2 \pi n+m \Omega$, where $m$ is a multiple of 3 ; for the unbalanced load, by contrast, all frequencies of the form $\omega_{0}+2 \pi n+m \Omega$ are present.

5. More efficient calculation of the input spectrum. The results of section 3 provide expressions for the input and output currents, and thus solve the problem posed at the start of this paper. However, each coefficient in the input current spectrum requires the evaluation of a doubly infinite sum, as in (3.12) and (3.14). Furthermore, these sums converge rather slowly. So we now describe a more efficient means of obtaining the input current spectrum for specific output impedances. It differs from the calculation in section 3 by taking advantage of the known impedances to perform the convolution integrals in (3.9) before the sum over switching times is 
evaluated. A consequence is that each Fourier coefficient will then require calculation of only a single infinite sum.

The explicit calculations outlined are for the case where each output load takes the form of a resistor and an inductor in series, so that the corresponding impedances are given by (4.2). The various complex impedances need not be equal between outputs $a, b$, and $c$. We shall discuss later how this method may be extended to the case of more general forms for the output impedances.

5.1. Illustration of transfer matrix calculation for purely resistive loads. We begin by considering purely resistive loads, with $L^{o}=0$. Although such loads are of limited practical interest, they are nevertheless useful to illustrate the following transfer matrix method. For a purely resistive load, the admittances of the three output lines are simply the constants $Y^{o}=1 / R^{o}$. This case is thus considerably easier to analyze than that of general impedance because a step output voltage of the sort described in section 3.2 produces an output current only while the voltage is switched on. Then, for example, the terms in the sum (3.7) vanish unless $n=m$ and we find that the diagonal terms in $Q$ take the form

$$
Q^{i i}(t)=\sum_{o=a, b, c} \frac{1}{R^{o}} F^{o i}(t) \mathrm{e}^{\mathrm{i} \omega_{0} t}, \quad i=A, B, \text { or } C .
$$

Note that the functions $F^{o i}(t)$ have been defined in (2.17). The off-diagonal terms in $Q$ necessarily vanish for resistive loads. To see this, consider, for example,

$$
Q^{A B}(t)=\sum_{o=a, b, c} \sum_{m, n} \frac{1}{R^{o}} \psi_{m, m+\alpha_{m}^{o}}(t) \psi_{n+\alpha_{n}^{o}, n+\beta_{n}^{o}}(t) .
$$

It is clear that the intervals $\left(m, m+\alpha_{m}^{o}\right)$ and $\left(n+\alpha_{n}^{o}, n+\beta_{n}^{o}\right)$ never overlap and hence $Q^{A B}=0$. A similar consideration shows that all other off-diagonal terms are zero.

The three input currents are then, using the results and notation of sections 2.3 and 2.5 ,

$$
\begin{aligned}
& i^{A}(t)=Q^{A A}(t)=\sum_{n m} Y_{m} X_{m}^{A}\left(x_{n m}\right) \mathrm{e}^{\mathrm{i} \Omega_{n m} t}, \\
& i^{B}(t)=p Q^{B B}(t)=\sum_{n m} p Y_{m} X_{m}^{B}\left(x_{n m}\right) \mathrm{e}^{\mathrm{i} \Omega_{n m} t}, \\
& i^{C}(t)=p^{2} Q^{C C}(t)=\sum_{n m} p^{2} Y_{m} X_{m}^{C}\left(x_{n m}\right) \mathrm{e}^{\mathrm{i} \Omega_{n m} t},
\end{aligned}
$$

where $x_{n m}=\omega_{n m}$ for regular sampling, and $x_{n m}=2 \pi n$ for natural sampling. Here

$$
Y_{m}=\sum_{o=a, b, c} \frac{\mathrm{e}^{\mathrm{i} m \delta^{o}}}{R^{o}}
$$

is an effective total output admittance, common to all three inputs.

These two simplifying elements of the matrix $Q$ (namely that $Q$ is a diagonal matrix, and that the diagonal elements are given by a single sum, as in (5.1)) follow from there being a purely resistive load. The key points are that an output voltage pulse produces a proportional output current pulse, and that after the voltage pulse the corresponding current drops immediately to zero. 
5.2. Frequency domain calculation for series resistor/inductor loads. We now consider the case of more general loads, with inductive as well as resistive elements, with output impedances given by (4.2). In this case, the double sum in (3.9) does not collapse to a single sum as it did in the purely resistive case and the calculation is more complex.

Before summing the series, let us consider in more detail the structure of the summand, whose general form is given in (3.4). For an inductive load with impedance $Z=R+i \omega L=i L(\omega-i \gamma)$, where $\gamma=R / L$ is the decay rate of transients in the current, we can write (3.4) more explicitly as

$$
\hat{\imath}_{t_{1}, t_{2}, t_{3}, t_{4}}(\omega)=\frac{1}{2 \pi \mathrm{i}} \int_{-\infty}^{\infty} \frac{\left(\mathrm{e}^{-\mathrm{i}\left(\omega-\omega^{\prime}\right) t_{2}}-\mathrm{e}^{-\mathrm{i}\left(\omega-\omega^{\prime}\right) t_{1}}\right)\left(\mathrm{e}^{-\mathrm{i}\left(\omega^{\prime}-\omega_{0}\right) t_{4}}-\mathrm{e}^{-\mathrm{i}\left(\omega^{\prime}-\omega_{0}\right) t_{3}}\right)}{L\left(\omega^{\prime}-\omega\right)\left(\omega^{\prime}-\mathrm{i} \gamma\right)\left(\omega^{\prime}-\omega_{0}\right)} \mathrm{d} \omega^{\prime},
$$

having substituted the explicit forms for $\hat{\psi}_{t_{1}, t_{2}}(\omega)$ and $\hat{\psi}_{t_{3}, t_{4}}(\omega)$ in the convolution integral. This is conveniently evaluated using the residue calculus. The denominator in the integrand has three zeroes, two on the real axis and one on the positive imaginary axis. The zeroes on the real axis are canceled by zeroes in the numerator and do not lead to poles in the total integrand. Since the integrand is analytic on the real axis, we may move the contour slightly off the real axis before beginning the calculation proper and the manner in which we do this will not affect the final result. This observation is relevant because we will evaluate the integral by expanding the numerator and considering terms individually. Although these individual terms have poles on the real axis, if we have deformed the contour away from these poles beforehand, the individual integrals are well defined. Furthermore, any contributions made by the poles on the real axis through the residue calculus must combine consistently and be independent of the initial contour deformation. The integral is therefore controlled by the pole at $\omega^{\prime}=i \gamma$. There are three cases to consider.

Case 1. Window $t_{1}, t_{2}$ precedes window $t_{3}, t_{4}$. In this case, where $t_{1}<t_{2}<t_{3}<t_{4}$, every term in the expanded numerator

$$
\begin{aligned}
& \left(\mathrm{e}^{-\mathrm{i}\left(\omega-\omega^{\prime}\right) t_{2}}-\mathrm{e}^{-\mathrm{i}\left(\omega-\omega^{\prime}\right) t_{1}}\right)\left(\mathrm{e}^{-\mathrm{i}\left(\omega^{\prime}-\omega_{0}\right) t_{4}}-\mathrm{e}^{-\mathrm{i}\left(\omega^{\prime}-\omega_{0}\right) t_{3}}\right) \\
& =\mathrm{e}^{-\mathrm{i} \omega t_{2}+\mathrm{i} \omega_{0} t_{4}+\mathrm{i} \omega^{\prime}\left(t_{2}-t_{4}\right)}-\mathrm{e}^{-\mathrm{i} \omega t_{2}+\mathrm{i} \omega_{0} t_{3}+\mathrm{i} \omega^{\prime}\left(t_{2}-t_{3}\right)} \\
& \quad-\mathrm{e}^{-\mathrm{i} \omega t_{1}+\mathrm{i} \omega_{0} t_{4}+\mathrm{i} \omega^{\prime}\left(t_{1}-t_{4}\right)}+\mathrm{e}^{-\mathrm{i} \omega t_{1}+\mathrm{i} \omega_{0} t_{3}+\mathrm{i} \omega^{\prime}\left(t_{1}-t_{3}\right)}
\end{aligned}
$$

decays exponentially as $\omega^{\prime}$ descends into the lower-half plane. The contour of integration can therefore be pushed downwards and, because there are no poles in the lower-half plane, the integral must vanish. We therefore necessarily have

$$
\hat{\imath}_{t_{1}, t_{2}, t_{3}, t_{4}}(\omega)=0
$$

in this case. Note that in the case of more complicated load impedances, causality demands that all of the zeroes of $Z\left(\omega^{\prime}\right)$ lie in the upper-half plane and the result (5.4) still holds. The result is obvious in the time domain because a driving voltage confined to the window $\left(t_{3}, t_{4}\right)$ produces no current for $t<t_{3}$, and any sampling window confined to this range must produce a null result.

Case 2. Window $t_{1}, t_{2}$ follows window $t_{3}, t_{4}$.

In this case, $t_{3}<t_{4}<t_{1}<t_{2}$. Let us assume that the integration contour has been moved slightly above the real axis before the calculation for individual terms in expansion (5.3) begins. Then, all of the terms on the right of (5.3) are exponentially 
decaying into the upper-half plane and we push the contour upwards, picking up a contribution only from the pole at $\omega^{\prime}=\mathrm{i} \gamma$. This yields

$$
\begin{aligned}
\hat{\imath}_{t_{1}, t_{2}, t_{3}, t_{4}}(\omega) & =\frac{\left(\mathrm{e}^{-\mathrm{i}(\omega-\mathrm{i} \gamma) t_{2}}-\mathrm{e}^{-\mathrm{i}(\omega-\mathrm{i} \gamma) t_{1}}\right)\left(\mathrm{e}^{\mathrm{i}\left(\omega_{0}-\mathrm{i} \gamma\right) t_{4}}-\mathrm{e}^{\mathrm{i}\left(\omega_{0}-\mathrm{i} \gamma\right) t_{3}}\right)}{L(\omega-\mathrm{i} \gamma)\left(\omega_{0}-\mathrm{i} \gamma\right)} \\
& =\frac{1}{L}\left(\frac{\mathrm{e}^{-\mathrm{i}(\omega-\mathrm{i} \gamma) t_{2}}-\mathrm{e}^{-\mathrm{i}(\omega-\mathrm{i} \gamma) t_{1}}}{-\mathrm{i}(\omega-\mathrm{i} \gamma)}\right)\left(\frac{\mathrm{e}^{\mathrm{i}\left(\omega_{0}-\mathrm{i} \gamma\right) t_{4}}-\mathrm{e}^{\mathrm{i}\left(\omega_{0}-\mathrm{i} \gamma\right) t_{3}}}{\mathrm{i}\left(\omega_{0}-\mathrm{i} \gamma\right)}\right) \\
& =\frac{1}{L} \hat{\psi}_{t_{1}, t_{2}}(\omega-\mathrm{i} \gamma) \hat{\psi}_{t_{3}, t_{4}}\left(-\omega_{0}+\mathrm{i} \gamma\right) .
\end{aligned}
$$

For more general load impedances, there would be a sum of such contributions, each term corresponding to a zero of $Z\left(\omega^{\prime}\right)$, or equivalently a decay rate of the system, weighted by residues of $1 / Z\left(\omega^{\prime}\right)$.

Case 3 . Window $t_{1}, t_{2}$ coincides with window $t_{3}, t_{4}$.

In this case, $t_{1}=t_{3}<t_{2}=t_{4}$. The calculation here is somewhat more complicated and requires a more careful consideration of the poles on the real axis. We forgo the details here and simply quote the result:

$$
\begin{aligned}
& \hat{\imath}_{t_{1}, t_{2}, t_{3}, t_{4}}(\omega)= \frac{1}{\mathrm{i} L\left(\omega_{0}-\mathrm{i} \gamma\right)}\left(\frac{\mathrm{e}^{-\mathrm{i}\left(\omega-\omega_{0}\right) t_{2}}-\mathrm{e}^{-\mathrm{i}\left(\omega-\omega_{0}\right) t_{1}}}{-\mathrm{i}\left(\omega-\omega_{0}\right)}\right) \\
&-\frac{\mathrm{e}^{\mathrm{i}\left(\omega_{0}-\mathrm{i} \gamma\right) t_{1}}}{\mathrm{i} L\left(\omega_{0}-\mathrm{i} \gamma\right)}\left(\frac{\mathrm{e}^{-\mathrm{i}(\omega-\mathrm{i} \gamma) t_{2}}-\mathrm{e}^{-\mathrm{i}(\omega-\mathrm{i} \gamma) t_{1}}}{-\mathrm{i}(\omega-\mathrm{i} \gamma)}\right) \\
&=\frac{1}{Z\left(\omega_{0}\right)}\left[\hat{\psi}_{t_{1}, t_{2}}\left(\omega-\omega_{0}\right)-\mathrm{e}^{\mathrm{i}\left(\omega_{0}-\mathrm{i} \gamma\right) t_{1}} \hat{\psi}_{t_{1}, t_{2}}(\omega-\mathrm{i} \gamma)\right],
\end{aligned}
$$

which is obtained by summing the contributions from the various poles for each of the terms in (5.3) and following some further algebraic manipulation.

So far we have established the forms of the individual terms in (3.9). It remains to perform the double sum over switching times in that equation. Once again we concentrate initially on the element $\hat{Q}^{B B}(\omega)$ and indicate later how the calculation is altered for other elements. In this case, the summands in (3.9) are of the form $\hat{\imath}_{t_{1}, t_{2}, t_{3}, t_{4}}(\omega)$, as calculated above, with $t_{1}=m+\alpha_{m}^{o}, t_{2}=m+\beta_{m}^{o}, t_{3}=n+\alpha_{n}^{o}$, $t_{4}=n+\beta_{n}^{o}$, and with loads that are output-specific. Let us denote by

$$
\gamma^{o}=R^{o} / L^{o}
$$

the decay rate of transients associated with output $o$. In view of (5.4), the summands vanish if $n<m$, so let us set $n=m+r$ for $r=0,1,2, \ldots$, and separate (3.9) into "diagonal" and "off-diagonal" contributions:

$$
\hat{Q}^{B B}(\omega)=\hat{Q}_{\text {diag }}^{B B}(\omega)+\hat{Q}_{\text {offdiag }}^{B B}(\omega),
$$

where

$$
\hat{Q}_{\text {diag }}^{B B}(\omega)=\sum_{o=a, b, c} \sum_{n=-\infty}^{\infty} \hat{\imath}_{n+\alpha_{n}^{o}, n+\beta_{n}^{o}, n+\alpha_{n}^{o}, n+\beta_{n}^{o}}(\omega)
$$

and

$$
\hat{Q}_{\text {offdiag }}^{B B}(\omega)=\sum_{o=a, b, c} \sum_{r=1}^{\infty} \sum_{n=-\infty}^{\infty} \hat{\imath}_{n+r+\alpha_{n+r}^{o}, n+r+\beta_{n+r}^{o}, n+\alpha_{n}^{o}, n+\beta_{n}^{o}}(\omega) .
$$


The diagonal contribution accounts for the terms with $r=0$ and corresponds to the case of coinciding windows given in (5.6). We expect these terms to dominate the total sum and so describe them first. We start with regular sampling (natural sampling is discussed at the end of this section). Then using the notation of section 2.3, we write

$$
\begin{aligned}
\sum_{n=-\infty}^{\infty} \hat{\psi}_{t_{1}, t_{2}}\left(\omega-\omega_{0}\right) & =\sum_{n=-\infty}^{\infty} \hat{\psi}_{n+\alpha_{n}^{o}, n+\beta_{n}^{o}}\left(\omega-\omega_{0}\right)=\hat{F}^{o B}\left(\omega-\omega_{0}\right) \\
& =2 \pi \sum_{n m} X_{m}^{B}\left(\omega_{n m}\right) \mathrm{e}^{m \mathrm{i} \delta^{o}} \delta\left(\omega-\Omega_{n m}\right)
\end{aligned}
$$

recalling that $\Omega_{n m}=\omega_{0}+\omega_{n m}=\omega_{0}+2 \pi n+m \Omega$. A similar calculation shows that

$$
\begin{aligned}
\sum_{n=-\infty}^{\infty} \mathrm{e}^{\mathrm{i}\left(\omega_{0}-\mathrm{i} \gamma^{o}\right) t_{1}} \hat{\psi}_{t_{1}, t_{2}}\left(\omega-\mathrm{i} \gamma^{o}\right) & =\sum_{n=-\infty}^{\infty} \mathrm{e}^{\mathrm{i}\left(\omega_{0}-\mathrm{i} \gamma^{o}\right)\left(n+\alpha_{n}^{o}\right)} \hat{\psi}_{n+\alpha_{n}^{o}, n+\beta_{n}^{o}}\left(\omega-\mathrm{i} \gamma^{o}\right) \\
& =\sum_{n=-\infty}^{\infty} \mathrm{e}^{-\mathrm{i} n\left(\omega-\omega_{0}\right)} \mathrm{e}^{\mathrm{i}\left(\omega_{0}-\mathrm{i} \gamma^{o}\right) \alpha_{n}^{o}} \hat{\psi}_{\alpha_{n}^{o}, \beta_{n}^{o}}\left(\omega-\mathrm{i} \gamma^{o}\right) \\
& =2 \pi \sum_{n m} Y_{m}^{B}\left(\omega_{n m},-\omega_{0}+\mathrm{i} \gamma^{o}\right) \mathrm{e}^{\mathrm{i} m \delta^{o}} \delta\left(\omega-\Omega_{n m}\right)
\end{aligned}
$$

where

$$
Y_{m}^{B}\left(\omega, \omega^{\prime}\right) \equiv \sum_{k=-\infty}^{\infty} X_{k}^{B}\left(\omega-\omega^{\prime}\right) C_{m-k}^{B}\left(\omega^{\prime}\right)
$$

and

$$
C_{m}^{B}(\omega)=\mathrm{e}^{-\mathrm{i} \omega / 3}(-\mathrm{i})^{m} \mathrm{~J}_{m}\left(\frac{2}{3} q \omega\right)
$$

Note that the expression defining $Y_{m}^{B}\left(\omega, \omega^{\prime}\right)$ can be summed using Graf's theorem [11]. However, leaving the definition of $Y_{m}^{B}\left(\omega, \omega^{\prime}\right)$ as a sum, as done here, has the advantage of admitting easier generalization to other diagonal terms and being simpler to write. We can therefore write

$$
\hat{Q}_{\text {diag }}^{B B}(\omega)=2 \pi \sum_{o=a, b, c} D_{n m}^{o, B} \mathrm{e}^{\mathrm{i} m \delta^{o}} \delta\left(\omega-\Omega_{n m}\right),
$$

where

$$
D_{n m}^{o, B}=\frac{1}{Z^{o}\left(\omega_{0}\right)}\left(X_{m}^{B}\left(\omega_{n m}\right)-Y_{m}^{B}\left(\omega_{n m},-\omega_{0}+\mathrm{i} \gamma^{o}\right)\right)
$$

There are similar diagonal contributions to $\hat{Q}^{A A}$ and $\hat{Q}^{C C}$, except that $C_{k}^{A}(\omega)$ and $C_{k}^{C}(\omega)$ have the alternative forms

$$
C_{m}^{A}(\omega)=\delta_{m 0}, \quad C_{m}^{C}(\omega)=\mathrm{e}^{-2 \mathrm{i} \omega / 3-\mathrm{i} m \pi / 3}(-\mathrm{i})^{m} \mathrm{~J}_{m}\left(\frac{2}{3} q \omega\right) .
$$

We next discuss off-diagonal contributions. In this case the summands are of the form given in (5.5) and 


$$
\begin{aligned}
\hat{Q}_{\text {nondiag }}^{B B}(\omega) & =\sum_{o=a, b, c} \sum_{r=1}^{\infty} \sum_{n=-\infty}^{\infty} \frac{1}{L^{o}} \hat{\psi}_{t_{1}, t_{2}}\left(\omega-\mathrm{i} \gamma^{o}\right) \hat{\psi}_{t_{3}, t_{4}}\left(-\omega_{0}+\mathrm{i} \gamma^{o}\right) \\
& =\sum_{o=a, b, c} \sum_{r=1}^{\infty} \sum_{n=-\infty}^{\infty} \frac{1}{L^{o}} \mathrm{e}^{-\mathrm{i} n\left(\omega-\omega_{0}\right)-\mathrm{i} r\left(\omega-\mathrm{i} \gamma^{o}\right)} \\
& \quad \times \hat{\psi}_{\alpha_{n+r}^{o}, \beta_{n+r}^{o}}\left(\omega-\mathrm{i} \gamma^{o}\right) \hat{\psi}_{\alpha_{n}^{o}, \beta_{n}^{o}}\left(-\omega_{0}+\mathrm{i} \gamma^{o}\right) \\
& =2 \pi \sum_{o=a, b, c} \sum_{n m} A_{n m}^{o, B B} \mathrm{e}^{\mathrm{i} m \delta^{o}} \delta\left(\omega-\Omega_{n m}\right),
\end{aligned}
$$

where (after some manipulation)

$$
A_{n m}^{o, B B}=\frac{1}{L^{o}} U_{m}^{B B}\left(\omega_{n m},-\omega_{0}+\mathrm{i} \gamma^{o}\right)
$$

and

$$
U_{m}^{B B}\left(\omega, \omega^{\prime}\right)=\sum_{k=-\infty}^{\infty} G_{1}\left(\omega-\omega^{\prime}-k \Omega\right) X_{k}^{B}\left(\omega-\omega^{\prime}\right) X_{m-k}^{B}\left(\omega^{\prime}\right)
$$

and

$$
G_{1}(\omega)=\sum_{r=1}^{\infty} \mathrm{e}^{-\mathrm{i} r \omega}=\frac{1}{\mathrm{e}^{\mathrm{i} \omega}-1} .
$$

The off-diagonal contributions to $\hat{Q}^{A A}$ and $\hat{Q}^{C C}$ are of the same form, with appropriate replacements for $X_{m}^{B}$.

If we now consider elements $\hat{Q}^{i j}(\omega)$ with $i \neq j$, we find that the appropriate intervals $\left(t_{1}, t_{2}\right)$ and $\left(t_{3}, t_{4}\right)$ never overlap and all summands are of the form given in (5.5). The calculation is very similar to that for $\hat{Q}_{\text {offdiag }}^{B B}(\omega)$ except that, when $i>j$, the sum over $r$ starts from $r=0$ rather than $r=1$ (here we adopt the convention that $C>B>A$ ). The result is the following generalization of (5.9):

$$
\hat{Q}^{i j}(\omega)=2 \pi \sum_{o=a, b, c} \sum_{n m} A_{n m}^{o, i j} \mathrm{e}^{\mathrm{i} m \delta^{o}} \delta\left(\omega-\Omega_{n m}\right),
$$

where

$$
A_{n m}^{o, i j}=\frac{1}{L^{o}} U_{m}^{i j}\left(\omega_{n m},-\omega_{0}+\mathrm{i} \gamma^{o}\right)
$$

and

$$
U_{m}^{i j}\left(\omega, \omega^{\prime}\right)=\sum_{k=-\infty}^{\infty} G^{i j}\left(\omega-\omega^{\prime}-k \Omega\right) X_{k}^{i}\left(\omega-\omega^{\prime}\right) X_{m-k}^{j}\left(\omega^{\prime}\right)
$$

and

$$
G^{i j}(\omega)= \begin{cases}\mathrm{e}^{\mathrm{i} \omega} G_{1}(\omega) & \text { if } i>j, \\ G_{1}(\omega) & \text { if } i<j .\end{cases}
$$

Again, the sum defining $U_{m}^{i j}\left(\omega, \omega^{\prime}\right)$ can be expressed alternatively using Graf's theorem but the form given is simpler to write. Note that if we set $i=j$, then (5.10) also describes the off-diagonal part of $\hat{Q}^{i i}$, if we take $G^{i i}(\omega)=G_{1}(\omega)$. 
The current in the input labeled by the subscript $i$ is (cf. (3.13))

$$
i^{i}(t)=Q^{i A}(t)+p Q^{i B}(t)+p^{2} Q^{i C}(t),
$$

where

$$
Q^{i j}(t)=\sum_{o=a, b, c} \sum_{n m}\left(D_{n m}^{o, i} \delta_{i j}+A_{n m}^{o, i j}\right) \mathrm{e}^{\mathrm{i} m \delta^{o}} \mathrm{e}^{\mathrm{i} \Omega_{n m} t} .
$$

Thus, from (5.12), the coefficient of each frequency component in the input current $i^{i}(t)$ requires (aside from sums over the three output lines) only a single infinite sum for computing each of the the terms $D_{n m}^{o, i}$ and $A_{n m}^{o, i j}$, as in (5.8) and (5.11).

Finally, we note also that a similar calculation is possible in the case of natural sampling. The answer in that case is similar, the main difference being that the functions $X_{m}^{i}, Y_{m}^{i}$, and $U_{m}^{i j}$ take different arguments when they are used in the calculation of the amplitudes $D_{n m}^{o, i}$ and $A_{n m}^{o, i j}$ (compare (2.20) with (2.31), for example).

6. Conclusions. We have shown how to compute the output voltage spectrum, and the output and input current spectra for an idealized matrix power converter, for general output loads. Our method provides a rather more direct alternative to the usual approach of Black's multiple Fourier series [2,3], and appears to be the first published calculation of the full spectrum. The mathematical expressions involved in the present calculations are considerably more compact than would be the equivalent expressions using Black's method. Despite its greater directness, however, our method still requires calculations that are rather algebraically involved. We have shown how reasonable assumptions about the form of the output loads - for example, if they are all series resistor-inductor loads - can be used for deriving more rapidly convergent expressions for the input currents (which are of particular significance since they provide an easily monitored diagnostic of the system). We note that the calculation in this paper can be adapted relatively easily to more general output impedances.

One potential practical upshot of our work is the following. In applications such as aeronautics, there are strict regulations regarding acceptable levels of the electromagnetic interference generated by high-frequency switching applications such as matrix converters. This paper provides, apparently for the first time, analytical expressions for the full frequency spectrum of voltages and currents. We therefore expect the formulas derived herein, and appropriate extensions of the methodology to more general cases (for example, a wider range of output impedances) to allow engineers to design matrix converters to satisfy mandatory restrictions on power quality without wasteful overspecification of the associated filters.

In the appendix, we illustrate how similar techniques can be adapted to more general switching protocols for the matrix converter. However, these introduce new frequencies into the spectrum, so the calculation is more involved.

The Fourier transform/Poisson resummation techniques applied here (with a judicious choice of the order in which the elements of the technique are applied, according to whether regular or natural sampling is used) may also be applied to other switching problems. Notable examples are the class-D audio amplifier, for which an analysis such as that given in this paper would lead to considerably more compact derivations of the spectrum than previously given $[3,8]$, and $\mathrm{DC}-\mathrm{AC}$ converters (inverters) [7].

Appendix. The modulation strategies considered in the main text are the simplest possible; in practice, more complicated strategies are used. Many of these will be amenable to a treatment similar to that described in this paper, but with increased 
algebraic complexity. In this appendix we illustrate some of the necessary modifications by calculating the output voltage spectrum for natural sampling using a hybrid Venturini modulation strategy. In this case, the switching times are determined by

$$
\begin{aligned}
& A^{o}(\tau)=\frac{1}{3}+\frac{2}{3} q\left[\theta \cos \left(\left(\omega_{1}-\omega_{0}\right) \tau+\delta^{o}\right)+(1-\theta) \cos \left(\left(\omega_{1}+\omega_{0}\right) \tau+\delta^{o}\right)\right], \\
& B^{o}(\tau)=\frac{2}{3}+\frac{2}{3} q\left[\theta \cos \left(\left(\omega_{1}-\omega_{0}\right) \tau+\delta^{o}-\frac{\pi}{3}\right)+(1-\theta) \cos \left(\left(\omega_{1}+\omega_{0}\right) \tau+\delta^{o}+\frac{\pi}{3}\right)\right],
\end{aligned}
$$

where $0 \leq \theta \leq 1$, rather than by (2.30). Notice that the case $\theta=1$ recovers (2.30). The case $\theta=1 / 2$ proves particularly straightforward to implement in practice [12].

The calculation of $F^{o i}(t)$ is now rather more involved, since

$$
\mathrm{e}^{-2 \pi n \mathrm{i} A^{o}(t)}=\mathrm{e}^{-2 \pi n \mathrm{i} / 3} \sum_{m=-\infty}^{\infty} \sum_{m^{\prime}=-\infty}^{\infty} \mathcal{C}_{m m^{\prime}} \mathrm{e}^{\mathrm{i}\left(m\left(\omega_{1}-\omega_{0}\right)+m^{\prime}\left(\omega_{1}+\omega_{0}\right)\right) t}
$$

and

$$
\mathrm{e}^{-2 \pi n \mathrm{i} B^{\circ}(t)}=\mathrm{e}^{-4 \pi n \mathrm{i} / 3} \sum_{m=-\infty}^{\infty} \sum_{m^{\prime}=-\infty}^{\infty} \mathcal{C}_{m m^{\prime}} \mathrm{e}^{\mathrm{i}\left(m\left(\omega_{1}-\omega_{0}\right)+m^{\prime}\left(\omega_{1}+\omega_{0}\right)\right) t} \mathrm{e}^{\mathrm{i}\left(m^{\prime}-m\right) \pi / 3},
$$

where

$$
\mathcal{C}_{m m^{\prime}}=(-\mathrm{i})^{m+m^{\prime}} \mathrm{J}_{m}\left(\frac{4}{3} n \pi q \theta\right) \mathrm{J}_{m^{\prime}}\left(\frac{4}{3} n \pi q(1-\theta)\right) \mathrm{e}^{\mathrm{i}\left(m+m^{\prime}\right) \delta^{\circ}} .
$$

Thus, in general, these quantities now involve additional frequencies beyond those present for the simpler case $\theta=1$.

Writing the output voltages as

$$
v^{o}(t)=\sum_{n m m^{\prime}} \tilde{V}_{n m m^{\prime}} \mathrm{e}^{\mathrm{i}\left(2 \pi n+\omega_{0}+m\left(\omega_{1}-\omega_{0}\right)+m^{\prime}\left(\omega_{1}+\omega_{0}\right)\right) t} \mathrm{e}^{\mathrm{i}\left(m+m^{\prime}\right) \delta^{o}},
$$

we have

$$
\tilde{V}_{n m m^{\prime}}=X_{m m^{\prime}}^{A}(2 \pi n)+p X_{m m^{\prime}}^{B}(2 \pi n)+p^{2} X_{m m^{\prime}}^{C}(2 \pi n),
$$

where

$$
\begin{aligned}
& X_{m m^{\prime}}^{A}(\omega)=\frac{(-\mathrm{i})^{m+m^{\prime}}}{-\mathrm{i} \omega}\left[\mathrm{e}^{-\mathrm{i} \omega / 3} \mathrm{~J}_{m}\left(\frac{2}{3} q \omega \theta\right) \mathrm{J}_{m^{\prime}}\left(\frac{2}{3} q \omega(1-\theta)\right)-\delta_{m 0} \delta_{m^{\prime} 0}\right], \\
& X_{m m^{\prime}}^{B}(\omega)=\frac{(-\mathrm{i})^{m+m^{\prime}}}{-\mathrm{i} \omega}\left[\mathrm{e}^{-2 \mathrm{i} \omega / 3+\mathrm{i}\left(m^{\prime}-m\right) \pi / 3}-\mathrm{e}^{-\mathrm{i} \omega / 3}\right] \mathrm{J}_{m}\left(\frac{2}{3} q \omega \theta\right) \mathrm{J}_{m^{\prime}}\left(\frac{2}{3} q \omega(1-\theta)\right), \\
& X_{m m^{\prime}}^{C}(\omega)=\frac{(-\mathrm{i})^{m+m^{\prime}}}{-\mathrm{i} \omega}\left[\mathrm{e}^{-\mathrm{i} \omega} \delta_{m 0} \delta_{m^{\prime} 0}-\mathrm{e}^{-2 \mathrm{i} \omega / 3+\mathrm{i}\left(m^{\prime}-m\right) \pi / 3} \mathrm{~J}_{m}\left(\frac{2}{3} q \omega \theta\right) \mathrm{J}_{m^{\prime}}\left(\frac{2}{3} q \omega(1-\theta)\right)\right] .
\end{aligned}
$$

Special consideration needs to be given to the values of $X_{m m^{\prime}}^{i}(0)$. We find that $X_{m m^{\prime}}^{i}(0)=0$ for $i=A, B$, or $C$, except in the following cases:

$$
X_{00}^{i}(0)=\frac{1}{3}, \quad i=A, B, \text { or } C
$$

and

$X_{0 \pm 1}^{A}(0)=\frac{1}{3} q(1-\theta), \quad X_{0 \pm 1}^{B}(0)=\frac{1}{3} q(1-\theta)\left(\mathrm{e}^{ \pm \mathrm{i} \pi / 3}-1\right), \quad X_{0 \pm 1}^{C}(0)=-\frac{1}{3} q(1-\theta) \mathrm{e}^{ \pm \mathrm{i} \pi / 3}$ 
and

$$
X_{ \pm 10}^{A}(0)=\frac{1}{3} q \theta, \quad X_{ \pm 10}^{B}(0)=\frac{1}{3} q \theta\left(\mathrm{e}^{\mp \mathrm{i} \pi / 3}-1\right), \quad X_{ \pm 10}^{C}(0)=-\frac{1}{3} q \theta \mathrm{e}^{\mp \mathrm{i} \pi / 3} .
$$

It then follows from (A.1) that for the contribution to the output voltages with $n=0$ we have $\tilde{V}_{00-1}=q(1-\theta)$ and $\tilde{V}_{010}=q \theta$, with $\tilde{V}_{0 m m^{\prime}}=0$ for all other choices of $m$ and $m^{\prime}$. Thus the corresponding contribution to the output voltages is

$$
v_{0}^{o}(t)=q \theta \mathrm{e}^{\mathrm{i} \omega_{1} t+\mathrm{i} \delta^{o}}+q(1-\theta) \mathrm{e}^{-\mathrm{i} \omega_{1} t-\mathrm{i} \delta^{o}} .
$$

Hence the physical output voltage, given by the real part of this expression, is, as for the simpler modulation strategy of section 2.5 , exactly the intended reference voltage $v_{\text {ref }}^{o}(t)$. A similar calculation can be undertaken for uniform sampling but is not described here.

\section{REFERENCES}

[1] A. Alesina And M. G. B. Venturini, Solid-state power conversion: A Fourier analysis approach to generalized transformer synthesis, IEEE Trans. Circuits and Systems, 28 (1981), pp. 319-330.

[2] W. R. Bennett, New results in the calculation of modulation products, Bell Syst. Tech. J., 12 (1933), pp. 228-243.

[3] H. S. Black, Modulation Theory, Van Nostrand, New York, 1953.

[4] S. R. Bowes, New sinusoidal pulsewidth-modulated inverter, Proc. IEEE, 122 (1975), pp. 12791285.

[5] J. C. Clare, L. Empringham, and P. W. Wheeler, The effects of sampling delays and non-ideal filtering on the performance of matrix converter modulation algorithms, in Proceedings of the Eighth International Conference on Power Electronics and Variable Speed Drives, London, 2000, pp. 29-34.

[6] S. M. Cox AND B. H. CANDY, Class-D audio amplifiers with negative feedback, SIAM J. Appl. Math., 66 (2005), pp. 468-488.

[7] D. G. Holmes And T. A. Lipo, Pulse Width Modulation for Power Converters: Principles and Practice, IEEE Press Ser. Power Engrg., IEEE Press, Piscataway, NJ, 2003.

[8] Z. Song AND D. V. SARwate, The frequency spectrum of pulse width modulated signals, Signal Processing, 83 (2003), pp. 2227-2258.

[9] M. Venturini, A new sine wave in, sine wave out conversion technique eliminates reactive elements, in Proceedings of the Powercon 7, San Diego, 1980, pp. E3_1-E3_15.

[10] M. Venturini And A. Alesina, The generalized transformer: A new bidirectional sinusoidal waveform frequency converter with continuously adjustable input power factor, in Proceedings of the IEEE PESC'80, Atlanta, 1980, pp. 242-252.

[11] G. N. Watson, A Treatise on the Theory of Bessel Functions, 2nd ed., Cambridge University Press, Cambridge, UK, 1944.

[12] P. W. Wheeler, J. Rodríguez, J. C. Clare, L. Empringham, and A. Weinstein, Matrix converters: A technology review, IEEE Trans. Industr. Electr., 49 (2002), pp. 276-288. 